\title{
p-Box: A new graph model
}

\author{
Mauricio Soto $\left.{ }^{1}\right|^{*}$ and Christopher Thraves Caro" $\|^{\mid}$
}

${ }^{1}$ DIM, Universidad de Chile, Chile

${ }^{2}$ GSyC, Universidad Rey Juan Carlos, Spain

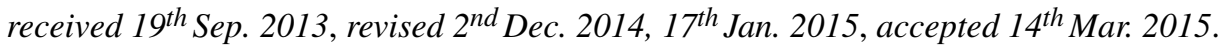

In this document, we study the scope of the following graph model: each vertex is assigned to a box in $\mathbb{R}^{d}$ and to a representative element that belongs to that box. Two vertices are connected by an edge if and only if its respective boxes contain the opposite representative element. We focus our study on the case where boxes (and therefore representative elements) associated to vertices are spread in $\mathbb{R}$. We give both, a combinatorial and an intersection characterization of the model. Based on these characterizations, we determine graph families that contain the model (e. g., boxicity 2 graphs) and others that the new model contains (e. g., rooted directed path). We also study the particular case where each representative element is the center of its respective box. In this particular case, we provide constructive representations for interval, block and outerplanar graphs. Finally, we show that the general and the particular model are not equivalent by constructing a graph family that separates the two cases.

Keywords: Graph theory, graph representation, disk graphs, intersection graphs, (max-)tolerance graphs, boxicity 2 graphs.

\section{Introduction}

A unit disk graph is the intersection graph of a family of unit disks in the Euclidean plane. That is, it is a graph with one vertex for each disk, and with an edge between two vertices if and only if the corresponding disks have non-empty intersection. An important application of unit disk graphs is in the area of sensor networks. Sensor networks are networks formed by sensor nodes, little devices deployed in a geographic area with monitor purposes. Sensors communicate with each other via a radio channel. Every sensor covers with its radio signal a communication area around it and two sensors communicate with each other when they are placed within each other's communication areas. In an ideal model, the communication area of a sensor is a circle. Therefore, in the same ideal model, if every sensor covers equally sized communication areas, the network formed by sensors is a unit disk graph. That explains why researchers have used unit disk graphs to represent sensor networks [FCFM09], or some variations [KWZ08].

\footnotetext{
*Email: mausoto@dim.uchile.cl. Gratefully acknowledges the support of CONICYT, Apoyo al retorno de investigadores desde el extranjero grant 82130059.

${ }^{\dagger}$ Email: cbthravesegsyc.es. Christopher Thraves Caro is supported by Spanish MICINN grant Juan de la Cierva, Comunidad de Madrid grant S2009TIC-1692 and Spanish MICINN grant TIN2008-06735-C02-01.
} 
Nevertheless, it is difficult to find such an ideal situation in a real deployment, mainly due to physical or geographical restrictions. For instance, when the deployment area is irregular, the communication area of a sensor might be shrunken in one direction due to an obstacle, while, in the opposite direction, the area is free of any obstacle. On the other hand, some sensors may have directional antennas which produce communication areas that are far from being a circle, or that place the sensor location far from the center of its communication area. Therefore, the existence of a communication link between two sensors is not determined by the distance between them, neither by the intersection of their communication areas. In fact, one has to be sure that the communication areas cover the opposite sensor.

Consequently, we propose a new graph family that aims to include the different topologies that may be created due to those restrictions. Consider a set $F$. Consider now a set $S \subseteq F$ and an element $p \in S$ as a representative element of $S$. Let us define a graph where each vertex corresponds to a pair $(S, p)$ and an edge between two vertices exists if and only if the set associated with a vertex contains the representative element of its fellow and vice versa. According to this definition, nonempty intersection between two sets is not enough to guarantee the existence of their corresponding edge. Moreover, when $F$ is a metric space, there is no positive distance between two representative elements that guarantees the existence of their corresponding edge. Therefore, this definition differs from unit disk graphs, as well as from other intersection models.

In this document, we consider the family induced by the above definition when sets are boxes in $\mathbb{R}^{d}$. We aim to understand the properties of such a graph family. We focus our study on the case where boxes and representative elements associated to vertices are spread in $\mathbb{R}$. We study the extent of this definition as a graph family. We provide an intersection model and a combinatorial characterization of the model. Additionally, we tackle the subfamily of graphs where all representative elements are the centers of their respective boxes.

\section{Definitions}

We consider graphs that are finite, connected, undirected, loopless and without parallel edges. For a graph $G=(V, E)$, we denote by $V(G)$ and $E(G)$ the set of vertices and edges, respectively. When the graph under consideration is clear, we use only $V$ and $E$. The edge $\{u, v\}$ is denoted by $u v$. If $u v \in E(G)$ we say that $v$ is a neighbor of $u$ and vice versa. The set of neighbors of $u$ is denoted by $\mathcal{N}(u)$. Additionally, the closed neighborhood of $u$ is defined as $\mathcal{N}[u]:=\mathcal{N}(u) \cup\{u\}$.

A box in $\mathbb{R}^{d}$ is the Cartesian product of $d$ closed intervals. A box $B$ is described as the set $B=$ $\left\{\left(x_{1}, x_{2}, \ldots, x_{d}\right) \in \mathbb{R}^{d}: L_{i} \leq x_{i} \leq R_{i}\right\}$, where $L_{i}$ and $R_{i}$ denote the extreme points of the interval in the $i$-th dimension. The center of a box $B$ as defined above is the point $\left(\left(L_{1}+R_{1}\right) / 2,\left(L_{2}+R_{2}\right) / 2, \ldots,\left(L_{d}+\right.\right.$ $\left.R_{d}\right) / 2$ ).

Definition 1 ( $p$-BoX-realization) A p-BoX-realization of a graph $G$ in $\mathbb{R}^{d}$ is a collection of pairs $\left\{\left(B_{v}, p_{v}\right): v \in V(G)\right\}$ where each vertex $v$ is associated to a box $B_{v} \in \mathbb{R}^{d}$ and to a representative element $p_{v} \in B_{v}$, such that:

$$
u v \in E(G) \Leftrightarrow\left(p_{v} \in B_{u}\right) \wedge\left(p_{u} \in B_{v}\right)
$$

A central $p$-BoX-realization or c-p-BoX-realization of a graph is a p-BoX-realization in which each representative element $p_{v}$ is the center of its box $B_{v}$. 
We denote by $p$-Box $(d)$ the set of graphs that admit a $p$-Box-realization in $\mathbb{R}^{d}$. The subset of $p$ $\operatorname{Box}(d)$ that contains the graphs that admit a $c-p$-BoX-realization in $\mathbb{R}^{d}$ is denoted by $c-p-\operatorname{Box}(d)$. For simplicity, in this document we use the notation $(c-) p$-BoX when we say something that concerns to both classes $c$ - $p$-BoX and $p$-BoX.

We mainly study the sets $p$ - $\operatorname{Box}(1)$ and $c-p$-Box(1). In this context, a box $B_{u}$ becomes an interval in $\mathbb{R}$ that we denote by its extreme points $[L(u), R(u)]$. Any $(c-) p$-Box $(1)$-realization can be modified in certain ways so that it maintains the graph it represents. For a given realization $\mathcal{R}=\left\{\left([L(u), R(u)], p_{u}\right)\right.$ : $u \in V(G)\}$, we define $\delta$-translation and $\sigma$-scaling of $\mathcal{R}$ as the realizations $\left\{\left([L(u)+\delta, R(u)+\delta], p_{u}+\delta\right)\right.$ : $u \in V(G)\}$ and $\left\{\left([\sigma \cdot L(u), \sigma \cdot R(u)], \sigma \cdot p_{u}\right): u \in V(G)\right\}$, respectively.

Any $(c-) p$-Box $(1)$-realization of a graph induces a natural ordering of its vertices following its representative elements, i.e., $v<u$ according to a $(c-) p$-Box(1)-realization if and only if $p_{v}<p_{u}$ in that $(c-) p$-Box $(1)$-realization. In order to properly define this order, each representative element must differ from each other. Nevertheless, it is easy to see that any $(c-) p$-Box(1)-realization can be modified to fulfill this property.

Given an ordering $\pi$ of the vertices of a graph $G$, we denote by $<_{\pi}$ the total order induced by $\pi$. That is, $u<_{\pi} v$ if $u$ appears before $v$ in $\pi$. The extreme vertices of an order $<_{\pi}$ are the vertices placed at the first and last position according to $<_{\pi}$. Given a vertex $u$, we denote by $\ell_{\pi}(u)$ and $\rho_{\pi}(u)$ the leftmost and rightmost neighbors of $u$ in the order, i.e., $\ell_{\pi}(u)=\left\{v \in \mathcal{N}[u]: v<_{\pi} w \forall w \in \mathcal{N}[u], w \neq v\right\}$ and $\rho_{\pi}(u)=\left\{v \in \mathcal{N}[u]: w<_{\pi} v \forall w \in \mathcal{N}[u], w \neq v\right\}$.

\section{Related work and our contributions}

We compare the introduced $p$-Box family of graphs with other graph classes. Therefore, we refer the reader to an excellent survey authored by Brandstädt et al. [BLS99] that contains a description of almost all graph families involved in this document. This survey also presents containment relations between classes, graphs that separate one class from another, and priceless information in this area. A second excellent book that we refer to the reader is authored by J. Spinrad [Spi03]. This book deals with efficient graph representation. For several graph classes, this book considers such questions as existence of good representations, algorithms for finding representations, questions of characterization in terms of representation, and how the representation affects the complexity of optimization problems.

There exists a vast amount of interesting literature related with the graph families that we mention in this document [MM99, Gol04, Rob69a, GT04]: geometric, outerplanar, interval, max-tolerance and boxicity 2 graphs. The study of intersection graphs dates back a long way. For instance, the fact that every graph can be represented as an intersection graph was proved by Marczewski and Sur in [SMS45] and by Erdös et al. in [EGP64]. Related with particular graph families, the notion of boxicity of a graph is introduced in [Rob69b]. On the other hand, the notion of book embedding of a graph is introduced in [BK79] where the authors present some first properties and relations with other invariants such as thickness, genus, and chromatic number. An intersection model for max-tolerance graphs is introduced in [KKLS06]. Such a model was of great utility in order to prove the NP-Hardness of recognition problem for max-tolerance graphs. Finally, the book [GT04] surveys results related with (max-)tolerance graphs.

The $p$-Box(1) family has been addressed very recently in parallel to our work via totally independent way. T. Hixon in his Master thesis [Hix13] studies the family of cyclic segment graphs. This family corresponds to the intersection graphs of segments that lie on lines tangent to a parabola and no two segments are parallel. In his thesis, Hixon also works on the subclass called hook graphs, in which all 
segments in the representation need to be tangent to the parabola. The author proves that a graph is a hook graph if and only if it is the intersection graph of a set of axis aligned rectangles in the plane such that the top left corner of each rectangle lies on a unique point on the line $x=y$. Such result is equivalent to the particular case $d=1$ of Theorem 1 in this document. The author also proves a combinatorial characterization for hook graphs which is equivalent to Theorem 2 in our work. This result has been also obtained independently by Correa et al. [CFS14] in the study of the gap between Minimum Hitting Size problem and Maximum Independent Set problem for the $p$-Box(1) family, and by Catanzaro et al. $\left[\mathrm{CCH}^{+}\right]$. Based on this characterization Hixon proves that interval, outerplanar, and 2directional orthogonal ray graphs (2DORG) are all hook graphs. We extend these results by proving that interval and outerplanar graphs are not only $p$-Box(1) but also $c-p$-Box(1) graphs. On the other hand, we extend the fact that interval graphs belong to $p$ - $\operatorname{Box}(1)$ by proving that a larger family, rooted directed path graphs, belongs to $p$-Box(1). Moreover, T. Hixon proves that in a hook graph two non-adjacent vertices cannot be connected by three induced disjoint paths of length larger than 4 . We prove indeed that such paths, in the case of $c-p$-Box(1), cannot be all of length 2 or longer. The author gives also polynomial algorithms for the Weighted Maximum Clique problem and Weighted Maximum Independent Set problem; and approximations for the Chromatic Number and Clique Covering Number. Finally, the $p$-Box(1) family has been independently addressed by Catanzaro et al. $\left[\mathrm{CCH}^{+}\right]$. In their work they characterize $p$ - $\operatorname{Box}(1)$ graphs as intersection graphs of axis-aligned L-shapes whose corner points form a line with negative slope. That result is a particular case of Theorem 1 They also show that the class of interval graphs belongs to $p$ - $\operatorname{Box}(1)$. As we have said, in this work we prove that a superclass of interval graphs, rooted directed tree graphs, belongs to $p$-Box(1). Finally, they observe that outerplanar graphs are a subclass of $p$ - $\operatorname{Box}(1)$. Nevertheless, their proof is completely different from ours.

\subsection{Our contributions}

The main contribution of this document is the study of the two graph families: $p$ - $\operatorname{Box}(d)$ and $c-p$ - $\operatorname{Box}(d)$. We study the one-dimensional version $(c-) p$-Box (1) of the families in which the position of the representative elements induce an order of the vertices.

- We give a characterization of both $p$ - $\operatorname{Box}(d)$ and $c-p$ - $\operatorname{Box}(d)$ families via an intersection model in Subsection 4.1. This result allows us to conclude that $p$-Box(1) graphs have boxicity 2 and that $c-p$ - $\operatorname{Box}(1)$ is a sub family of max-tolerance graphs.

- We give a characterization of the $p$-Box(1) family via a combinatorial characterization of the possible orders of its vertices in any $p$-Box(1)-realization in Subsection 4.2 .

- We give a construction of $p$-BOX(1)-realizations for ROOTED DIRECTED PATH graphs, and $c-p$ Box(1)-realizations for INTERVAL, BLOCK and OUterPlanAR graphs in Subsection 4.2 and Section 5 , respectively.

- Finally, in Section 6, we show differences between families $p$-Box(1) and $c-p$-Box(1), proving that in the first case two non-adjacent vertices cannot be connected via three disjoint paths with edge-length 4 or longer. While, in the second case, two non-adjacent vertices cannot be connected via three disjoint paths with edge-length 2 or longer. 


\section{Characterizations for $p$-Box graphs}

In this section, we show that $p$ - $\operatorname{Box}(d)$ graphs can be represented by an intersection model. Besides, we give a combinatorial characterization for the set of graphs that admit a $p$-Box(1)-realization. From these characterizations, we obtain containment relations with well known graph classes.

\subsection{Intersection graph characterization for $p$-Box graphs}

We show in this subsection that graphs in the $p$ - $\operatorname{Box}(d)$ class can be represented as the intersection graph of boxes in $\mathbb{R}^{2 d}$.

Theorem 1 A graph $G$ belongs to $p$ - $\operatorname{Box}(d)$ if and only if $G$ is the intersection graph of boxes in $\mathbb{R}^{2 d}$, where each box can be described as $\times_{i=1}^{d}\left(\left[p_{i}, R_{i}\right] \times\left[-p_{i},-L_{i}\right]\right)$ with $p_{i}, R_{i}, L_{i}>0$ for all $i \in\{1, \ldots, d\}$.

Proof: Let $G$ be a graph that belongs to $p$ - $\operatorname{Box}(d)$. Consider a realization $\left\{\left(B_{v}, p_{v}\right)\right\}_{v \in V}$ of $G$, where $B_{v}=\times_{i=1}^{d}\left[L_{i}(v), R_{i}(v)\right]$ and $p_{v}=\left(p_{(v, 1)}, p_{(v, 2)}, \ldots, p_{(v, d)}\right)$. W.1.o.g, assume $L_{i}(v)>0$ for all $v \in V$ and $i \in\{1, \ldots, d\}$. For each $v \in V$, we define the box $B_{(v, i)}^{\prime}$ in $\mathbb{R}^{2}$ as $\left[p_{(v, i)}, R_{i}(v)\right] \times\left[-p_{(v, i)},-L_{i}(v)\right]$. Finally, let $S_{v}$ be the $2 d$-dimensional box defined as the Cartesian product of boxes $B_{(v, i)}^{\prime}$, that is $S_{v}=$ $\times_{i=1}^{d}\left(\left[p_{(v, i)}, R_{i}(v)\right] \times\left[-p_{(v, i)},-L_{i}(v)\right]\right)$.

For two vertices $u, v \in V$, the edge $u v \in E$ if and only if for each $i \in\{1 \ldots, d\}, p(u, i) \in$ $\left[L_{i}(v), R_{i}(v)\right]$ and $p_{(v, i)} \in\left[L_{i}(u), R_{i}(u)\right]$. Notice that if the intervals $\left[p_{(u, i)}, R_{i}(u)\right]$ and $\left[p_{(v, i)}, R_{i}(v)\right]$ are disjoint, then we either have $R_{i}(u)<p_{(v, i)}$ or $R_{i}(v)<p(u, i)$, implying $p_{(v, i)} \notin\left[L_{i}(u), R_{i}(u)\right]$ or $p_{(u, i)} \notin\left[L_{i}(v), R_{i}(v)\right]$ respectively. Similarly, one can easily verify that if the intervals $\left[-p_{(u, i)},-L_{i}(u)\right]$ and $\left[-p_{(v, i)},-L_{i}(v)\right]$ are disjoint, equivalently the intervals $\left[L_{i}(u), p_{(u, i)}\right]$ and $\left[L_{i}(v), p_{(v, i)}\right]$ are disjoint, then also it should be the case that either $p_{(u, i)} \notin\left[L_{i}(v), R_{i}(v)\right]$ or $p_{(v, i)} \notin\left[L_{i}(u), R_{i}(u)\right]$. It can also be easily verified that if $\left[p_{(u, i)}, R_{i}(u)\right] \cap\left[p_{(v, i)}, R_{i}(v)\right] \neq \varnothing$ and $\left[-p_{(u, i)},-L_{i}(u)\right] \cap\left[-p_{(v, i)},-L_{i}(v)\right] \neq \varnothing$, then we have both $p_{(u, i)} \in\left[L_{i}(v), R_{i}(v)\right]$ and $p_{(v, i)} \in\left[L_{i}(u), R_{i}(u)\right]$. Therefore, $u v \in E$ if and only if for each $i \in\{1, \ldots, d\},\left[p_{(u, i)}, R_{i}(u)\right] \cap\left[p_{(v, i)}, R_{i}(v)\right] \neq \varnothing$ and $\left[-p_{(u, i)},-L_{i}(u)\right] \cap\left[-p_{(v, i)},-L_{i}(v)\right] \neq \varnothing$, or equivalently, $B_{(u, i)}^{\prime} \cap B_{(v, i)}^{\prime} \neq \varnothing$. Hence vertices $u$ and $v$ are adjacent if and only if $S_{u} \cap S_{v} \neq \varnothing$.

Conversely, let $H$ be the intersection graph of boxes in $\mathbb{R}^{2 d}$, where the box associated to vertex $v \in$ $V(H)$ is of the form $\times_{i=1}^{d}\left(\left[p_{(v, i)}, R_{i}(v)\right] \times\left[-p_{(v, i)},-L_{i}(v)\right]\right)$, with $p_{(v, i)}, R_{i}(v), L_{i}(v)>0$ for all $i \in$ $\{1, \ldots, d\}$. On the other hand, let $G$ be the graph induced by the $p$-BoX-realization $\left\{\left(B_{v}, p_{v}\right)\right\}_{v \in V}$, where $B_{v}=\times_{i=1}^{d}\left[L_{i}(v), R_{i}(v)\right]$ and $p_{v}=\left(p_{(v, 1)}, p_{(v, 2)}, \ldots, p_{(v, d)}\right)$. We shall show that $u v \in E(H)$ if and only if $u v \in E(G)$. Hence, we conclude that $H$ belongs to $p$-Box $(d)$.

Let $u$ and $v$ be two vertices of $H$. On the one hand, if $u v \in E(H)$ their respective boxes intersect. Therefore, for each $i \in\{1, \ldots, d\},\left[p_{(u, i)}, R_{i}(u)\right] \cap\left[p_{(v, i)}, R_{i}(v)\right] \neq \varnothing$ and $\left[-p_{(u, i)},-L_{i}(u)\right] \cap$ $\left[-p_{(v, i)},-L_{i}(v)\right] \neq \varnothing$. Hence, it holds that $p_{(u, i)} \in\left[L_{i}(v), R_{i}(v)\right]$ and $p_{(v, i)} \in\left[L_{i}(u), R_{i}(u)\right]$. Therefore, it holds that $u v \in E(G)$. On the other hand, if $u v \notin E(H)$ then their respective boxes do not intersect. Thus, for each $i \in\{1, \ldots, d\}$ it holds that either the intervals $\left[p_{(u, i)}, R_{i}(u)\right]$ and $\left[p_{(v, i)}, R_{i}(v)\right]$ are disjoint, or the intervals $\left[-p_{(u, i)},-L_{i}(u)\right]$ and $\left[-p_{(v, i)},-L_{i}(v)\right]$ are disjoint. By our previous discussion, we can conclude that for each $i \in\{1, \ldots, d\}$ it holds that either $p_{(u, i)} \notin\left[L_{i}(v), R_{i}(v)\right]$ or $p_{(v, i)} \notin\left[L_{i}(u), R_{i}(u)\right]$. Hence, it also holds that $u v \notin E(G)$. In conclusion, the edge $u v \in E(H)$ if and only if $u v \in E(G)$. Hence, the graph $H$ belongs to $p-\operatorname{Box}(d)$.

For the one-dimensional case $(d=1)$, Theorem 1 states that $p$-Box $(1)$ graphs correspond exactly to the intersection graphs of boxes in $\mathbb{R}^{2}$ with its left-lower corner lying on the diagonal $\mathcal{L}: x+y=0$ (an 

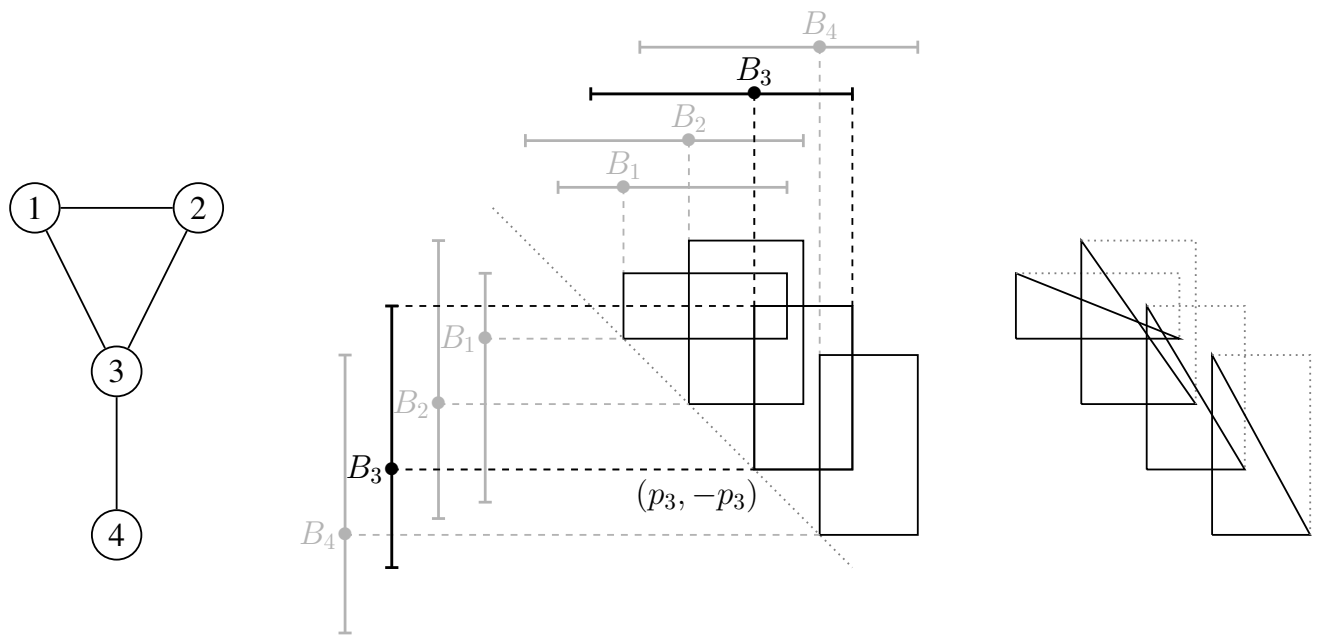

Fig. 1: An example of a graph in $p$-Box(1) (left) with its intersection model with boxes (center) and triangles (right hand side).

example is shown in Figure 11. On the other hand, a graph is said to have boxicity 2 if it has an intersection model consisting of axis-parallel boxes (i.e. rectangles) in $\mathbb{R}^{2}$ [Rob69b]. Therefore, we can conclude the following corollary.

Corollary 1 Let $\operatorname{BOXICITY}(2)$ be the set of graphs with boxicity 2. Thus p-BoX(1) $\subset \operatorname{BOXICITY}(2)$.

Kaufmann et al. in [KKLS06] proved that max-tolerance graphs correspond to the class of intersection of isosceles, axis parallel, right triangles (or lower halves of a square). A different representation of $p$ Box(1) graphs can be obtained by keeping the left lower half of the boxes in the intersection model (an example is shown in Figure 1). Particularly, when this intersection model is applied to $c-p$-Box $(1)$ graphs, we obtain an intersection model of isosceles, axis parallel, right triangles (or lower halves of a square). Therefore, the following corollary holds.

Corollary 2 Let MAX-TOLERANCE be the set of max-tolerance graphs. Thus c-p-BOX(1) $\subset$ MAXTOLERANCE.

\subsection{A combinatorial characterization for the $p$-Box(1) family}

We recall that any $p$-Box $(1)$-realization of a graph induces a natural ordering of its vertices by considering their respective representative elements. This ordering needs to have different representative elements in order to be totally defined. Nevertheless, it is easy to see that any $(c-) p$-Box $(1)$-realization can be modified to fulfill this property.

Definition 2 (R-order) Given a graph $G$ that belongs to $p$-Box(1) and a p-Box(1)-realization $\mathcal{R}$ of $G$ such that all representative elements are different. The $\mathcal{R}$-order of the set $V$, denoted by $<_{\mathcal{R}}$, is the total order induced by the representative elements. That is, for any pair of vertices $u$ and $v$ :

$$
u<_{\mathcal{R}} v \Leftrightarrow p_{u}<p_{v}
$$




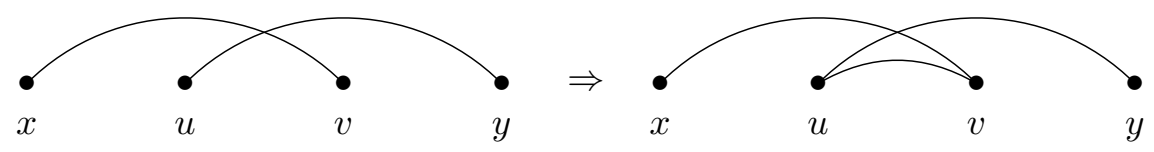

Fig. 2: Graphic representation of the four point condition for $p$-Box(1).

Consider an $\mathcal{R}$-order of a graph $G$ and two vertices $u<_{\mathcal{R}} v$ in $V$. If vertex $u$ has a neighbor $y$ after $v$ $\left(v<_{\mathcal{R}} y\right)$ and $v$ has a neighbor $x$ before $u\left(x<_{\mathcal{R}} u\right)$. Then, vertices $u$ and $v$ are mutually contained in its corresponding intervals. Thus, vertices $u$ and $v$ must be connected. Indeed, this property characterizes graphs that belong to the set $p$-Box(1). Therefore, we introduce the following definition for any ordering of the set of vertices of a graph.

Definition 3 Given a graph $G=(V, E)$ and an order $<_{\pi}$ of its set of vertices. We say that $<_{\pi}$ satisfies the four point condition for $p$ - $\operatorname{BOX}(1)$ if and only if for every quadruplet of vertices $x, u, v, y$, it holds:

$$
\text { If } x<_{\pi} u<_{\pi} v<_{\pi} y \text { and } x v, u y \in E \Rightarrow u v \in E .
$$

Figure 2 shows a graphic representation of the four point condition for $p$ - $\mathrm{Box}(1)$.

We prove that for any graph $G$ the existence of an ordering of its set of vertices that satisfies the four point condition for $p$ - $\operatorname{Box}(1)$ is necessary and sufficient to decide if $G$ belongs to $p$ - $\operatorname{Box}(1)$.

Theorem 2 A graph $G$ belongs to $p$-Box(1) if and only if there exists an ordering of its set of vertices that satisfies the four point condition for $p$ - $\mathrm{BOX}(1)$.

Proof: As we have seen previously, given any $p$-Box(1)-realization $\mathcal{R}$ of $G$, the four point condition is necessarily satisfied by the $\mathcal{R}$-order of the vertex set $V$. For the converse, let $<_{\pi}$ be any ordering of the vertices of $G$ which satisfies the four point condition.

Let $\mathcal{R}_{\pi}$ be a realization constructed in the following way: representative elements $p_{v}$ are embedded in $\mathbb{R}$ arbitrarily but respecting the order $<_{\pi}$. For each $v \in V$, we define $B_{v}$ as the interval covering from the leftmost to the rightmost neighbors of $v$ according to $<_{\pi}$, that is $B_{v}=\left[p_{\ell_{\pi}(v)}, p_{\rho_{\pi}(v)}\right]$.

In order to verify that $\mathcal{R}_{\pi}$ is a $p$ - $\operatorname{Box}(1)$-realization of $G$, consider vertices $u, v \in V$ with $u<_{\pi} v$. If $u v \in E$, then by definition of $\mathcal{R}_{\pi}$, it holds that $p_{u} \in B_{v}$ and $p_{v} \in B_{u}$. On the other hand, if $u v \notin E$, we have either $u<_{\pi} \ell_{\pi}(v)$ or $\rho_{\pi}(u)<_{\pi} v$, because if that is not the case, then we have $\ell_{\pi}(v)<_{\pi} u<_{\pi} v<_{\pi} \rho_{\pi}(u)$, implying that $<_{\pi}$ violates the four point condition. This implies that either $p_{u} \notin B_{v}$ or $p_{v} \notin B_{u}$. This shows that $\mathcal{R}_{\pi}$ is a valid $p$ - $\operatorname{Box}(1)$-realization of $G$.

Note that the above construction allows us to place the representative elements of the vertices in the integers ranging from 1 to $n$. Hence, any $p$-Box(1) graph can be represented as the collection of boxes $B_{v}=\left[l_{v}, r_{v}\right]$ and representative elements $p_{v}$, for all $v \in V$, where $l_{v}, r_{v}$ and $p_{v}$ are all integers ranging from 1 to $n$. On the other hand, in any $p$-Box(1)-realization, adjacency between two vertices $u$ and $v$ can be tested by performing four operations in order to check $p_{u} \in\left[l_{v}, r_{v}\right]$ and $p_{v} \in\left[l_{u}, r_{u}\right]$. Therefore, we can conclude that the following remark holds.

Remark 1 The family of graphs p-Box(1) admits an implicit representation as defined in [Spi03], which is: an implicit representation of a graph $G$ is defined as a representation of $G$ that assigns $O(\log n)$ bits to 
each vertex, such that there is an adjacency testing algorithm that decides adjacency between two vertices $u$ and $v$ based only on the bits stored at vertices $u$ and $v$.

\subsection{Applications of the four point condition}

The four point condition is a useful tool to recognize graph families that belong to the set $p$ - $\operatorname{Box}(1)$ as well as families that do not belong to it. We now present three graph families that belong to $p$-Box(1), for which we show the existence of an ordering that satisfies the four point condition.

We start with rooted directed path graphs. A graph is a rooted directed path graph (also known as directed path graphs) if it has an intersection model consisting of directed paths in a rooted directed tree, where every arc is oriented from the root to the leaves. Figure 3 shows an example of an intersection model with directed paths on a rooted directed tree and its corresponding intersection graph.
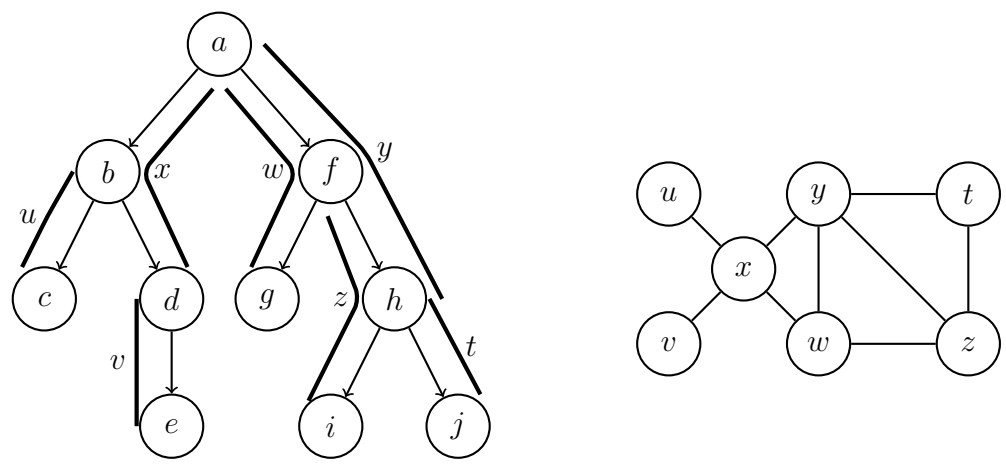

Fig. 3: At the right hand side, this figure shows an example of a rooted directed path graph. At the left hand side, this figure shows a rooted directed path representation of the graph. An inverse DFS on that tree is $(j i h g f e d c b a)$ inducing the following order of the vertices of the graph: $(t z y w v x u)$.

Let $G=(V, E)$ be a rooted directed path graph and $T=(K, F)$ be a rooted directed tree with an intersection model of $G$ consisting of directed paths in $T$. For each $v$ in $V$, let us denote by $K_{v}$ the vertex set of the directed path in $T$ corresponding to the vertex $v \in V$. Note that for every $v, K_{v}$ is a subset of $K$. We order $K$, the vertex set of $T$, using an inverse DFS ordering, i.e., first order $K$ according to a DFS (cf. [Gol04]), and then invert the ordering. Let us denote by $\pi: K \rightarrow\{1,2, \ldots,|K|\}$ the permutation given by the ordering of $K$, i.e., $\pi(k)=i$ when $k$ is the $i$-th vertex in the inverse DFS ordering. We define as well the following notation: $\pi\left(K_{v}\right)=\left\{\pi(k): k \in K_{v}\right\}$. An ordering $<_{I D F S}$ of the vertex set $V$ is called an IDFS ordering if it is obtained according to the minimum value of $\pi\left(K_{v}\right)$ where ties can be broken arbitrarily, i.e., $u<_{I D F S} v \Rightarrow \min \pi\left(K_{u}\right) \leq \min \pi\left(K_{v}\right)$.

Note that for any directed edge $(a, b) \in F$, we will have $\pi(b)<\pi(a)$ as any DFS will have to visit $a$, the parent of $b$ in $T$, before visiting $b$. This, combined with the fact that for any $u \in V, K_{u}$ is a directed path in $T$, gives us the following observation.

Observation 1 Let $i, k \in\{1,2, \ldots,|K|\}$ such that $i<k$ and $i, k \in \pi\left(K_{u}\right)$ for some $u \in V$. If for every $j$ such that $i<j<k$, we have $j \notin \pi\left(K_{u}\right)$, then $\pi^{-1}(i) \pi^{-1}(k) \in F$ (is an edge of $T$ ).

It can be easily seen that a DFS ordering $<^{\prime}$ of the vertex set $K$ of $T$ satisfies the following property for any $a, b, c \in K:$ if $a<^{\prime} b<^{\prime} c$ and $a c \in F$, then there is a directed path in $T$ from $a$ to $b$. Note that 
this also means that there can be no directed path in $T$ that contains both $b$ and some vertex $d$ such that $c \leq^{\prime} d$. Therefore, for a vertex $u \in V(G)$, it is not possible that $K_{u}$ contains both $b$ and some vertex $d$ such that $c \leq^{\prime} d$. Translating this to the IDFS ordering given by $\pi$, we have the following observation.

Observation 2 Let $i, j, k \in\{1,2, \ldots,|K|\}$ such that $i<j<k$ and $\pi^{-1}(i) \pi^{-1}(k) \in F$. Then for any $l \leq i$ and any vertex $u \in V$, we have $\{l, j\} \nsubseteq \mathbb{I}\left(K_{u}\right)$.

Lemma 1 Let $u, v \in V$. There does not exist $i, j, k, l \in\{1,2, \ldots,|K|\}$ such that $i \leq j<k<l, i, k \in$ $\pi\left(K_{u}\right), j, l \in \pi\left(K_{v}\right)$ and $k \notin \pi\left(K_{v}\right)$.

Proof: Suppose that such $i, j, k$ and $l$ exist. Let $k_{1}=\max \left\{q \in \pi\left(K_{v}\right): q<k\right\}$ and let $k_{2}=\min \{q \in$ $\left.\pi\left(K_{v}\right): q>k\right\}$. Clearly, we know that $k_{1}$ and $k_{2}$ exist as we have $j, l \in \pi\left(K_{v}\right)$ with $j<k<l$. This also tells us that $k_{1} \geq j$ and $k_{2} \leq l$. From Observation 1, we have $\pi^{-1}\left(k_{1}\right) \pi^{-1}\left(k_{2}\right) \in F$. Since we also have $i \leq k_{1}<k<k_{2}$, and $i, k \in \pi\left(K_{u}\right)$, we have a contradiction to Observation 2 . This concludes the proof.

Lemma 2 Let $x, u, v \in V$ be three vertices such that $x<_{I D F S} u<_{I D F S} v, x v \in E$ and $u v \notin E$. Then for every $j \in \pi\left(K_{u}\right)$, it holds that $j<\min \pi\left(K_{v}\right)$.

Proof: Suppose for the sake of contradiction that there exists a $j \in \pi\left(K_{u}\right)$ such that $j \geq \min \pi\left(K_{v}\right)$. As $K_{u} \cap K_{v}=\emptyset$, we know that $j \notin \pi\left(K_{v}\right)$, which also implies that $j>\min \left(K_{v}\right)$. We can deduce that $\max \pi\left(K_{v}\right)<j$, as otherwise, $\min \pi\left(K_{u}\right)<\min \pi\left(K_{v}\right)<j<\max \pi\left(K_{v}\right)$ would violate Lemma 1 for $u, v$. Therefore, we have $\min \pi\left(K_{u}\right)<\min \pi\left(K_{v}\right) \leq \max \pi\left(K_{v}\right)<j$. Now, since $K_{x} \cap K_{v} \neq \emptyset$, there exists some index $i \in \pi\left(K_{x}\right) \cap \pi\left(K_{v}\right)$. Thus, $\min \pi\left(K_{v}\right) \leq i \leq \max \pi\left(K_{v}\right)$. As $\pi\left(K_{u}\right) \cap \pi\left(K_{v}\right)=\emptyset$, we have $i \notin \pi\left(K_{u}\right)$. We now have $\min \pi\left(K_{x}\right) \leq \min \pi\left(K_{u}\right)<i<j$. But this violates Lemma 1 for $x, u$.

Corollary 3 ROOTED DIRECTED PATH graphs belong to the class p-BOX(1).

Proof: As before, let $G=(V, E)$ be a rooted directed path graph and $T=(K, F)$ be the underlying rooted directed tree for the intersection model of $G$. We shall use the notation $\pi,<_{I D F S}$, and $K_{u}, \pi\left(K_{u}\right)$ for $u \in V$ as defined before for the rest of this proof. We claim that $<_{I D F S}$ satisfies the four point condition, and hence $G$ is a $p$-Box(1) graph. Suppose for sake of contradiction that there exist four vertices $x<_{I D F S} u<_{I D F S} v<_{I D F S} y$ such that $x v, u y \in E$ but $u v \notin E$. From Lemma2, we know that $\max \pi\left(K_{u}\right)<\min \pi\left(K_{v}\right)$. Since $\pi\left(K_{u}\right) \cap \pi\left(K_{y}\right) \neq \emptyset$, this means that $\min \pi\left(K_{y}\right)<\min \pi\left(K_{v}\right)$, which contradicts the fact that $v<_{I D F S} y$.

\section{Corollary 4 OUterplanaR graphs belong to p-BOX (1).}

Proof: In order to prove that outerplanar graphs belong to $p$-Box(1), let us recall the definition of page embedding of a graph (cf. [BK79]). A $k$-page embedding, or book embedding, of a graph $G$ consists in a linear ordering of the vertices of $G$ which are drawn on a line (the spine of the book) together with a partition of the edges into $k$ pages such that two edges in the same page do not cross. The pagenumber of a graph is the smallest $k$ for which the graph has a $k$-page embedding. In [Bil92], Bilski proved that outerplanar graphs are exactly the graphs with pagenumber one. Therefore, for any outerplanar graph 
there exists an ordering of its vertices in which the edges do not cross. Such an ordering satisfies the four point condition for $p$-Box(1).

In contrast to the previous corollary, the four point condition helps as well to discard a graph from the $p$-Box(1) set.

Corollary 5 Let $G$ be a graph such that all pairs of vertices $u, v \in V$ have at least two non adjacent common neighbors. Then $G$ does not belong to $p$-Box(1).

Proof: The proof is by contradiction. Let us assume that there exists a graph $G$ that belongs to $p$-Box(1) such that all pairs of vertices $u, v \in V$ have at least two non adjacent common neighbors. In order to reach the contradiction, we give four vertices in $V$ that do not satisfy the four point condition for $p$-Box(1). Let $\mathcal{R}$ be a $p$-Box(1)-realization for $G$. Consider the two extreme vertices of $\mathcal{R}$, say vertices $x$ and $y$. There exist two vertices $u$ and $v$ such that $u v \notin E$ and $\{x u, x v, u y, v y\} \subseteq E$. Now, for any order of vertices $u$ and $v$, it holds that the quadruplet $x, u, v, y$ does not satisfy the four point condition for $p$-Box(1).

\section{Subclasses of $c-p$-Box $(1)$}

In this section, we establish the relation between the $c-p$-Box(1) family and other well-known graph families. Particularly, we enhance the result by Catanzaro et al. $\left[\mathrm{CCH}^{+}\right]$by showing that interval and outerplanar graphs belong not only to $p$-Box(1) but also to $c-p$-Box(1).

Theorem 3 The set of INTERVAL graphs is a subset of c-p-BOX(1).

Proof: Let $G$ be an interval graph. In [Ola91] Olariu proves that for any interval graph there exists an ordering $<_{\pi}$ of its vertex set $V$ such that for all triplets $u, v, w \in V$ with $u<_{\pi} v<_{\pi} w$ and $u w \in E$ then $u v \in E$. Moreover, this order can be obtained in linear time. Consider such an ordering for the vertex set $V$. For the sake of simplicity, we relabel vertices in $V$ from 1 to $n$ according to the ordering $<_{\pi}$. In the following, for a vertex $i \in V$, we denote $\ell_{\pi}(i)$ by $\ell(i)$ and $\rho_{\pi}(i)$ by $\rho(i)$.

We construct a $c-p$-Box $(1)$-realization $\left\{[L(i), R(i)], p_{i}\right\}_{i \in V}$ of $G$ greedily. At the $i$-th step, we include the vertex $i$ in the $c-p$-Box(1)-realization. The inclusion is performed in such a way that, at the end of the step $i$, it holds, for all $j, k, w$ in $\{1, \ldots, i\}$, that:
1. $p_{k-1}<p_{k}$
3. $L(j)<p_{\ell(j)}$
2. $\rho(j)<_{\pi} \rho(k) \Rightarrow R(j)<R(k)$.
4. $\rho(k)<_{\pi} j \Leftrightarrow R(k)<p_{j}$.

Condition 1 ensures that representative elements are placed according to order $<_{\pi}$. Condition 2 ensures that right extremes of intervals are in the same order as the values of $\rho(\cdot)$. Finally, conditions 3 and 4 guarantee that the partial realization at the end of step $i$ corresponds to the subgraph induced by vertices $1,2, \ldots, i$. Thus, at the end of the construction a $c-p$ - $\operatorname{Box}(1)$-realization of $G$ is obtained.

At the first step, vertex 1 is included so that $p_{1}=0$ and $[L(1), R(1)]=[-1,1]$. At the end of the first step all conditions are satisfied. Let us suppose that all conditions hold at the end of the step $i-1$. We include vertex $i$ in the $c-p$-Box(1)-realization in two phases:

- First, we set the position of representative element $p_{i}$ respecting conditions 1 and 4 That is, the representative element is placed after $p_{i-1}$ and it is contained only by intervals associated to its previous neighbors. 
- Second, we set the interval associated to $i$ such that it contains the representative elements of all its previous neighbors, according to condition 3 Finally, we modify, if necessary, the interval of previous vertices in order to satisfy conditions 2

For the first phase we remark that if two vertices $j, k$ have labels smaller than $i$ and $j \notin \mathcal{N}(i) \wedge k \in \mathcal{N}(i)$ then $\rho(j)<i \leq \rho(k)$. Therefore, by condition 2 , we have that $R(j)<R(k)$. Thus, by defining $L=$ $\max \{R(j): j \notin \mathcal{N}(i)\}$ and $R=\min \{R(k): k \in \mathcal{N}(i)\}$, it holds that $L<R$. Notice that in between $L$ and $R$ there might exist some representative elements. Hence, by setting $p_{i}$ as $\left(\max \left\{p_{i-1}, L\right\}+R\right) / 2$, conditions 1 and 4 hold and first phase is concluded.

In order to set the extremes of interval $B_{i}$, let us define $P_{i}=\{j<i: \rho(j)<\rho(i)\}$, the set of all vertices having its last neighbor before the last neighbor of $i$. We recall that condition 2 requires that $R(j)<R(i)$ for all vertex $j$ in $P_{i}$. If $R^{\prime}$ denotes the $\max \left\{R(j): j \in P_{i}\right\}$ then it must hold that $R^{\prime}<R(i)$. On the other hand, the interval $B_{i}$ must contain $p_{\ell(i)}$ so that condition 3 is satisfied. Then, let us define $r_{i}$ as $\max \left\{p_{i}-p_{\ell(i)}, R^{\prime}-p_{i}\right\}+1$. We set $L(i)=p_{i}-r_{i}$ and $R(i)=p_{i}+r_{i}$ so that all conditions are satisfied for vertices in $P_{i} \cup\{i\}$. However, condition 2, does not necessarily hold for vertices in $\{1,2, \ldots, i-1\} \backslash P_{i}$. To overcome this problem, we extend the intervals of the vertices in $\{1,2, \ldots, i-1\} \backslash P_{i}$ by $2 r_{i}$. That is, we re-define $B_{j}$ for $j \in\{1,2, \ldots, i-1\} \backslash P_{i}$ as $\left[L(j)-r_{i}, R(j)+r_{i}\right]$. Thus, since $R(i)=p_{i}+r_{i}<R(j)+r_{i}$ condition 2 is satisfied for all vertices in $\{1,2, \ldots, i\}$. It is easy to verify that the other three conditions are also satisfied for all the vertices after this operation.

The rest of the section aims to prove that OUTERPLANAR graphs belong to $c-p$-Box(1). We first show that cycles belong to $c-p$-Box(1). Moreover, we show that any realization of a cycle has a specific structure. Secondly, we construct a procedure to combine biconnected components and show how to "glue" a cycle to a (c-)p-Box(1) graph by an edge.

Lemma 3 Let $C_{n}$ be a cycle of length $n$, then $C_{n}$ belongs to c-p-Box(1). Furthermore, let $\mathcal{R}$ be a p$\operatorname{Box}(1)$-realization of $C_{n}$ and $\pi$ be the permutation induced by $<_{\mathcal{R}}$. Then, there exists a clockwise (or anticlockwise) labeling $l: V \rightarrow\{1,2, \ldots, n\}$ such that:

(a) Vertices $l^{-1}(1)$ and $l^{-1}(n)$ are the extreme vertices of the realization, i.e., $\pi\left(l^{-1}(1)\right)=1 \wedge$ $\pi\left(l^{-1}(n)\right)=n$. Therefore, extreme vertices of $\mathcal{R}$ are neighbors.

(b) For all $u \in V,|l(u)-\pi(u)| \leq 1$.

(c) If $\mathcal{R}$ is a c-p-Box(1)-realization then for all $u \in V, l(u)=\pi(u)$.

Proof: Let $C_{n}$ be a cycle. We prove that $C_{n}$ belongs to $c-p$-Box(1) by constructing an appropriate realization. Let us label the vertex set $V$ clockwise starting in an arbitrary vertex. We associate each vertex labelled $i \in\{2, \ldots, n-1\}$ with the interval $[i-1, i+1]$ and the representative element $p_{i}=i$. Extreme vertices labelled 1 and $n$ are assigned to pairs (interval, representative element) $([2-n, n], 1)$ and $([1,2 n-1], n)$, respectively. It is easy to check that the previous defined realization is a $c-p$-Box $(1)$ realization for $C_{n}$.

In order to prove the second part of the lemma, consider a $p$-Box(1)-realization $\mathcal{R}$ of the cycle $C_{n}$. If $n=3$ the representative elements are always in a clockwise or anticlockwise order. The labeling given by this order is the clockwise or anticlockwise labeling that satisfies conditions (a), (b), and (c). 
Assume then that $n>3$. We define a clockwise (or anticlockwise) labeling $l$ of $V$ as follows: (1) the vertex with label 1 has the minimum value of $p_{u}$, i.e., $\left(\pi \circ l^{-1}(1)=1\right)$ and, (2) the vertex with label 2 is the neighbor of 1 with the smaller position in the order: $\pi \circ l^{-1}(2)<\pi \circ l^{-1}(n)$.

Condition (a) is proved by contradiction. Note that by definition $l^{-1}(1)$ is an extreme vertex. Hence, assume that $l^{-1}(n)$ is not an extreme vertex. Define $w$ as follows: $p_{l^{-1}(n)}<p_{w}$ and $l(w) \leq l\left(w^{\prime}\right)$ for all $w^{\prime}$ such that $p_{l^{-1}(n)}<p_{w^{\prime}}$. By the definition of the labeling, it holds that $l(w)>2$, moreover $w$ has a neighbor placed between the vertices with labels 1 and $n$, which we denote by $v$. We conclude that the quadruplet $l^{-1}(1)<_{\mathcal{R}} v<_{\mathcal{R}} l^{-1}(n)<_{\mathcal{R}} w$ the violates four point condition, which is a contradiction. Hence, the vertex $l^{-1}(n)$ is an extreme vertex. Since $l$ is a clockwise (or anticlockwise) labeling, vertices $l^{-1}(1)$ and $l^{-1}(n)$ are neighbors.

We prove (b) and (c) greedily. First, let us introduce some definitions. We say that a vertex $u$ satisfies the pre-condition if for all $v$ such that $p_{v}<p_{u}$ it holds that $l(v)<\pi(u)$. Clearly, extreme vertices satisfy the pre-condition. Let $w$ be a vertex that satisfies the pre-condition but such that $l(w) \neq \pi(w)$. Then it must hold that $l(w)>\pi(w)$. Let us denote by $v$ the vertex with label $\pi(w)-1$. By definition of $w$, it holds that $\pi(w)<n$ and therefore, $l(v)<n-1$. We denote by $x$ the vertex with label $\pi(w)$, which is clearly a neighbor of $v$. Note that by definition of $w$, we now have $v<_{\mathcal{R}} w<_{\mathcal{R}} x$. Let $w^{\prime}$ be the vertex in between $v$ and $x$ with the maximum label. Clearly, $w^{\prime}$ has the highest label of all vertices before $x$ in $<_{\mathcal{R}}$ and since $l\left(w^{\prime}\right)<n$, it has one neighbor $y$ (with label $l\left(w^{\prime}\right)+1$ ) such that $x<_{\mathcal{R}} y$. We now have $v<_{\mathcal{R}} w^{\prime}<_{\mathcal{R}} x<_{\mathcal{R}} y$, and by the four point condition, it must be true that $w^{\prime}$ and $x$ are neighbors. From this, we have $l\left(w^{\prime}\right)=l(x)+1=l(v)+2$, and since every vertex between $v$ and $x$ has label higher than $v$, we can conclude that there is exactly one vertex between $v$ and $x$ in the ordering $<_{\mathcal{R}}$, which also means that $w=w^{\prime}$. Additionally, the vertex immediately after $x$, that is, in the position $\pi(x)+1=\pi(w)+2$, satisfies the pre-condition.

As we state before, extreme vertices satisfy the pre-condition. Let $w$ be the first vertex according to $<_{\mathcal{R}}$ such that $l(w) \neq \pi(w)$. By definition, $w$ satisfies the pre-condition. Thus, by the previous discussion, we have that $l(w)=\pi(w)+1$. Furthermore, the next vertex in the ordering, say $x$, has label $l(w)-1$ and then $l(w)-\pi(w)=1 \wedge l(x)-\pi(x)=-1$. Moreover, the next vertex in the ordering must satisfy the pre-condition. By iterating over vertices according to the order $<_{\mathcal{R}}$, we verify that Condition (b) holds. Finally, consider the case when $\mathcal{R}$ is a $c-p$ - $\operatorname{Box}(1)$-realization. Let $v<_{\mathcal{R}} w<_{\mathcal{R}} x<_{\mathcal{R}} y$ be the quadruplet previously constructed, where $l(y)=l(w)+1$. If $p_{x}$ is placed in the left half of the interval $\left[p_{v}, p_{y}\right]$ then $v w \in E$, otherwise $x y \in E$ which yields a contradiction. Thus, for all vertices in the $c-p$-Box $(1)$-realization $l(w)=\pi(w)$.

Definition 4 (Safe vertex) Let $G$ be a graph in (c-)p-Box(1). We say that a vertex $v \in V$ is safe in a $(c-) p$-Box $(1)$-realization $\mathcal{R}=\left\{\left(B_{u}, p_{u}\right)\right\}_{u \in V(G)}$ of $G$ if it holds that:

$$
v \in B_{w} \text { if and only if } v=w \vee v w \in E(G) .
$$

Moreover, we say that a vertex $v \in V$ is safe in $G$ if there exists a $(c-) p-\operatorname{Box}(1)$-realization $\mathcal{R}$ of $G$ such that $v$ is safe in $\mathcal{R}$.

A safe vertex allows the union of two different biconnected components. This important property comes from the fact that in a realization where a vertex $v$ is safe, the interval $B_{v}$ can be extended as much as required without modifying the original graph. 

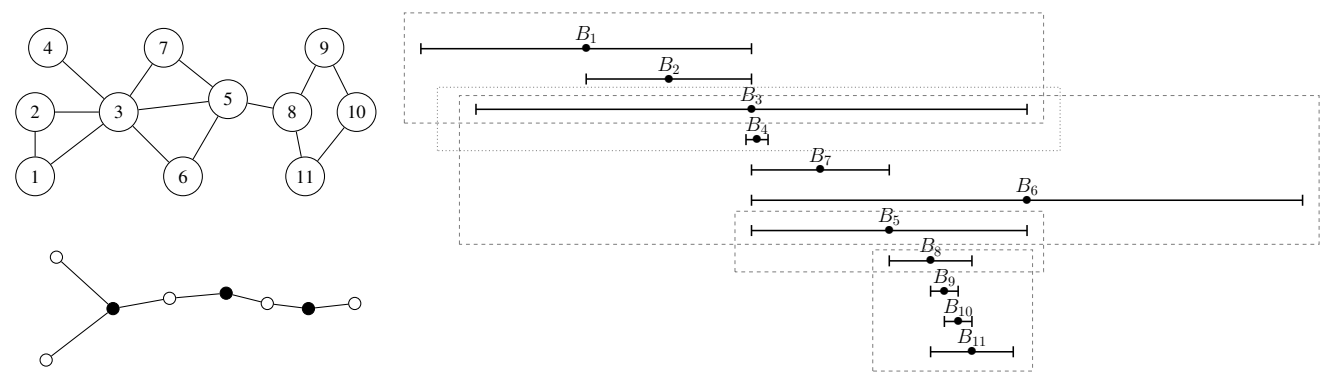

Fig. 4: This figure shows a graph (top left) and its block tree (bottom left). In the block tree representation, white vertices represent maximal biconnected components, while black vertices represent cut-vertices. Right hand side figure shows a $c-p$-Box(1)-realization of the graph according to the construction of Theorem 5 Dashed blocks represent the maximal biconnected components of the graph.

Lemma 4 Consider two graphs $G_{1}, G_{2} \in(c-)$ - $\operatorname{Box}(1)$ and two vertices $w_{1} \in V\left(G_{1}\right)$ and $w_{2} \in V\left(G_{2}\right)$. Let $G$ be the graph obtained by identifying $w_{1}$ and $w_{2}$. If $w_{2}$ is safe in $G_{2}$, then it holds that $G \in(c-) p$ $\operatorname{Box}(1)$.

Proof: Consider three graphs $G_{1}, G_{2}$ and $G$, and two vertices $w_{1}$ and $w_{2}$ defined as in the statement of the Lemma. Consider two $(c-)$ - $\operatorname{Box}(1)$-realizations $\mathcal{R}_{1}=\left\{\left(B_{u}, p_{u}\right)\right\}_{u \in V\left(G_{1}\right)}$ and $\mathcal{R}_{2}=\left\{\left(B_{u}, p_{u}\right)\right\}_{u \in V\left(G_{2}\right)}$ of $G_{1}$ and $G_{2}$, respectively, such that $w_{2}$ is safe in $\mathcal{R}_{2}$. We denote by $\Delta$ the minimum distance between any two representative elements in $\mathcal{R}_{1}$, that is $\Delta=\min _{u, v \in V\left(G_{1}\right)}\left\{\left|p_{u}-p_{v}\right|\right\}$. Let $B$ be an interval such that $\cup_{v \in V\left(G_{2}\right)}\left\{B_{v}\right\} \subseteq B$ and denote by $L$ its length. We construct the realization $\mathcal{R}_{2}^{\prime}=\left\{\left(B_{v}^{\prime}, p_{v}^{\prime}\right)\right\}_{v \in V\left(G_{2}\right)}$ from $\mathcal{R}_{2}$ by the following procedure:

- apply a $\left(-p_{w_{2}}\right)$-translation in order to place the representative element of $w_{2}$ in the origin,

- scale the realization by a factor $\Delta /(2 L)$,

- perform a $\left(p_{w_{1}}\right)$-translation in order to make the representative elements of $w_{1}$ and $w_{2}$ coincide.

Let $B_{w}$ be the interval with center in $p_{w_{1}}$ and of length equal to the maximum between $B_{w_{1}}$ and $B_{w_{2}}^{\prime}$. Then, let us define $\mathcal{R}=\mathcal{R}_{1} \cup \mathcal{R}_{2}^{\prime} \backslash\left\{\left(B_{w_{1}}, p_{w_{1}}\right),\left(B_{w_{2}}^{\prime}, p_{w_{2}}^{\prime}\right)\right\} \cup\left(B_{w}, p_{w}\right)$. We see that $\mathcal{R}$ is a $(c-) p$ Box (1) realization for $G$. In fact, all edges $u v \in E\left(G_{1}\right) \cup E\left(G_{2}\right)$ are induced by $\mathcal{R}$. Furthermore by the definition of $\mathcal{R}_{2}^{\prime}$ and the fact that $w_{2}$ is safe, no new edges are generated by $\mathcal{R}$.

Given a graph $G$, the block tree of $G$ is the graph having two types of vertices: blocks and cut-vertices (cf. [BM07]). A block vertex represents a maximal biconnected component of $G$ while cut-vertices are the articulation points between blocks. The edges of the block tree join blocks with cut-vertices. A block is adjacent to a cut-vertex if the block contains the cut-vertex. Figure 4 shows an example of a graph and its block tree.

Theorem 4 Let $G$ be a connected graph and $T$ be its block tree. If all maximal biconnected components of $G$ belong to (c-)p-BOX(1) and $T$ can be rooted such that every cut-vertex is safe in its children, then $G$ belongs to $(c-) p-\operatorname{Box}(1)$. 
Proof: The proof follows directly from Lemma 4 by adding biconnected components of $G$ in a breadthfirst traversal BFS (cf. [Gol04]) order of $T$. Figure 4 shows an example of this construction.

The previous result allows us to constructively obtain a realization of a graph by gluing the realization of its biconnected components. As a consequence, we obtain the following corollary.

Corollary 6 BLOCK graphs, graphs in which all biconnected components induce a clique, belong to $c-p-\operatorname{Box}(1)$.

An analogous result to Lemma 4 can be obtained to identify an edge in a cycle with an edge in a different realization:

Lemma 5 Let $H$ be a graph in c-p-Box(1) and $C_{n}$ a cycle of length $n$. Consider two edges $u^{\prime} v^{\prime} \in E(H)$ and $u v \in E\left(C_{n}\right)$ such that the representative elements of $u^{\prime}$ and $v^{\prime}$ are consecutive in a c-p-Box(1)realization of $H$. Let $G$ be the graph obtained by identifying uv and $u^{\prime} v^{\prime}$. Then, $G \in c-p-\operatorname{Box}(1)$.

Proof: Let $\mathcal{R}^{\prime}$ be a $c-p$-Box(1)-realization of $H$ in which $u^{\prime}$ and $v^{\prime}$ are consecutive. Let $\mathcal{R}$ be the $c$ $p$-Box(1)-realization of $C_{n}$ constructed according to the procedure described in the proof of Lemma 3 where $u$ and $v$ are the extreme vertices of the realization $\mathcal{R}$. We perform a scaling and a translation of $\mathcal{R}$ so that the positions of the representative elements of $u$ and $v$ coincide with those of $u^{\prime}$ and $v^{\prime}$ in $\mathcal{R}^{\prime}$. One may also need to do a horizontal reflection of $\mathcal{R}$ if the representative elements of $u$ and $v$ do not occur in the same order in both $\mathcal{R}$ and $\mathcal{R}^{\prime}$. The realization $\mathcal{R} \cup \mathcal{R}^{\prime} \backslash\left\{\left(B_{u}, u\right),\left(B_{v}, v\right)\right\}$ is a $c-p$-Box $(1)$-realization for the graph $G$. Notice that all vertices that were safe in $\mathcal{R}^{\prime}$ (including possibly $u^{\prime}$ and/or $v^{\prime}$ ) are also safe in the new realization as the interval for every vertex that came from $C_{n}$, other than $u$ and $v$, lies entirely between $p_{u^{\prime}}$ and $p_{v^{\prime}}$.

Theorem 5 The set of OUTERPLANAR graphs is a subset of c-p-BOX(1).

Proof: Let $G$ be an outerplanar graph. We shall show that for every maximal biconnected component $H$ of $G$ and a vertex $u$ in it, there exists a $c-p-\operatorname{Box}(1)$-realization of $H$ in which $u$ is safe. Then, by Theorem 4, we will be done. Since $G$ is an outerplanar graph, $H$ is either a single edge or a dissection of a convex polygon. If $H$ is a single edge, then it is easy to see that $H$ has a $c-p$-Box(1)-realization in which both its vertices are safe. Therefore, let us assume that $H$ is a dissection of a convex polygon, and $u \in V(H)$. Let $D$ be the weak dual of $H$, that is, the graph whose vertices correspond to the bounded faces of $H$ and two vertices are neighbors if and only if they share an edge in $H$ (Figure 5 shows a graph and its weak dual). As $H$ is a dissection of a convex polygon, $D$ is a tree. This means that we can construct $H$ iteratively following a breadth-first-search of $D$ starting at any vertex of $D$. One step of this procedure will be as follows. Suppose we are processing vertex $x$ of the tree $D$ at this step. Let $H^{\prime}$ be the subgraph of $H$ that we have constructed so far. In this step, we glue the cycle represented by $x$ to $H^{\prime}$ along the unique edge that is common to both $H^{\prime}$ and the cycle represented by $x$ (it is the edge that is common to the cycle represented by $x$ and its parent in the BFS). By applying Lemma 5 to each step of this construction, we can conclude that $H$ has a $c-p$-Box(1)-realization. Moreover, we can start the BFS of $D$ at a vertex corresponding to a face that contains $u$ to ensure that $u$ is safe in the $c-p$-Box(1)-realization of $H$ that we obtain through the application of Lemma 5 Thus, for any maximal biconnected component $H$ of $G$, and for any vertex $u \in V(H)$, there is a $c-p$ - $\operatorname{Box}(1)$-realization in which $u$ is safe. This completes the proof. 

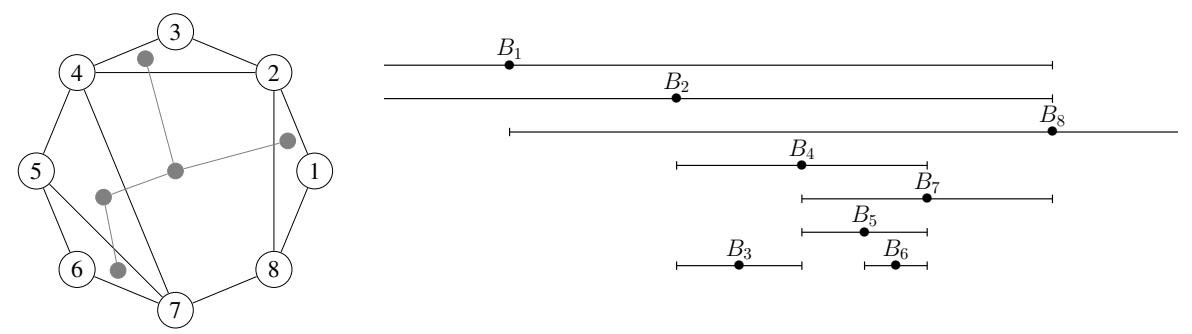

Fig. 5: The dissections of a convex polygon outerplanar graph with its weak dual (left hand size) and a construction of its $c-p$-Box(1)-realization according to Theorem 5 .
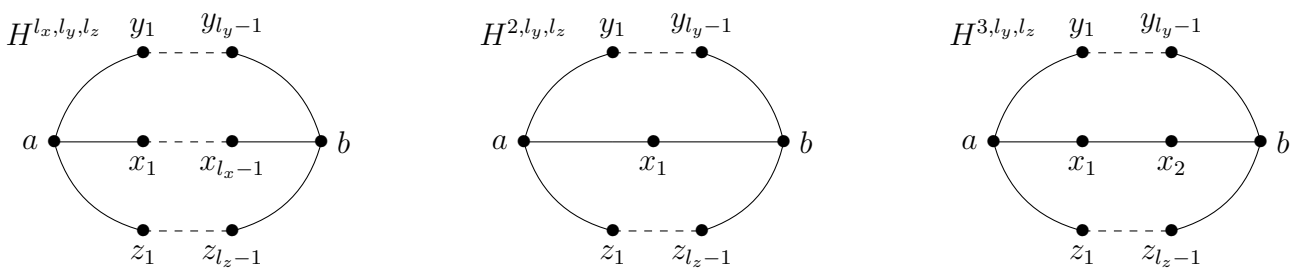

Fig. 6: This figure shows, from left to right, a graphic representation of (a general) $H^{l_{x}, l_{y}, l_{z}}$, and the particular cases of $H^{2, l_{y}, l_{z}}$ and $H^{3, l_{y}, l_{z}}$.

\section{Differences between $p$ - $\operatorname{Box}(1)$ and $c-p$ - $\operatorname{Box}(1)$}

In this section, we show the difference between $p$-Box(1) and $c-p$-Box(1) via graphs that belong to $p$ $\operatorname{Box}(1)$ but which do not belong to $c-p$-Box(1). We start with a remark upon the fact that the property of being part of $p$ - $\operatorname{Box}(1)$ or $c-p$ - $\operatorname{Box}(1)$ is hereditary, i.e., if a graph $G$ belongs to $(c-) p$ - $\operatorname{Box}(1)$ then every induced subgraph of $G$ also belongs to (c-)p-Box(1). Indeed, if a graph $G$ has a $(c-) p$-Box(1)-realization then the same realization is also a (c-)p-Box(1)-realization for every induced subgraph of $G$ when the corresponding vertices are deleted. From the hereditary property, we define a graph $G$ that does not belong to $(c-) p$-Box (1) as minimal with respect to $(c-) p$ - $\operatorname{Box}(1)$ if and only if every proper induced subgraph of $G$ does belong to $(c-) p$-Box(1). All graphs introduced here that separate $p$-Box(1) and $c-p$-Box(1) are minimal with respect to $c-p-\operatorname{Box}(1)$ and they are based in the following two definitions.

Definition 5 Let $H^{l_{x}, l_{y}, l_{z}}$ be a finite graph that consists of two not neighboring vertices, say vertices a and $b$, together with three vertex disjoint paths that connect vertex a with vertex $b$. The three paths that connect vertex $a$ with vertex $b$ follow: path $X=\left\{a=x_{0}, x_{1}, x_{2}, \ldots, x_{l_{x}-1}, x_{l_{x}}=b\right\}$, path $Y=\{a=$ $\left.y_{0}, y_{1}, y_{2}, \ldots, y_{l_{y}-1}, y_{l_{y}}=b\right\}$ and path $Z=\left\{a=z_{0}, z_{1}, z_{2}, \ldots, z_{l_{z}-1}, z_{l_{z}}=b\right\}$, where the edge-length of the paths, denoted by $l_{x}, l_{y}$, and $l_{z}$, are larger or equal than 2 . A graphic representation of $H^{l_{x}, l_{y}, l_{z}}$ is shown in Figure 6

Given a cycle $C=u_{1} u_{2} \ldots u_{k} u_{1}$, we say that a linear order $<$ of $V(C)$ respects $C$ if it is of the form $u_{i}<u_{i+1}<\cdots<u_{k}<u_{1}<u_{2}<\cdots<u_{i-1}$ or $u_{i}<u_{i-1}<\cdots<u_{1}<u_{k}<u_{k-1}<\cdots<u_{i+1}$, for some $i \in\{1, \ldots, k\}$. Given a linear order $<$ that respects $C$, any linear order obtained by restricting $<$ to a subset of $V(C)$ is said to partially respect $C$. 

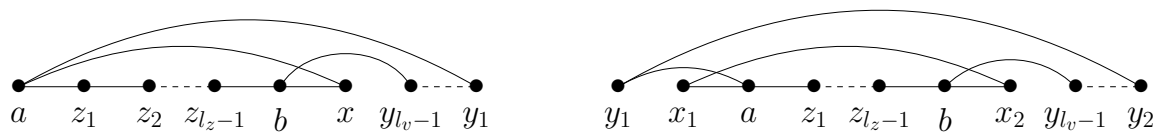

Fig. 7: This figure shows a graphic representation of $H^{2, l_{y}, l_{z}}$ and $H^{3, l_{y}, l_{z}}$ where the order of the vertices satisfies the four point condition.

Remark 2 Let $G$ be a graph and $\mathcal{R}$ be a p-Box(1)-realization of $G$. Consider an independent set $I$ in $G$. Let $C$ be an induced cycle in $G$. From Lemma 3. we can say that the linear order $<_{\mathcal{R}}$ restricted to $C \cap I$ partially respects $C$.

Lemma 6 Any $H^{l_{x}, l_{y}, l_{z}}$ graph such that $l_{z} \geq l_{y} \geq l_{x} \geq 4$ does not belong to $p$-Box $(1)$.

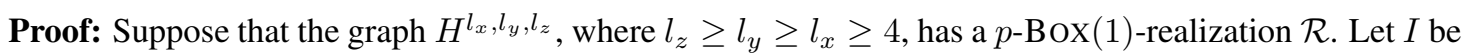
the independent set $a, b, x_{2}, y_{2}, z_{2}$. Define the cycles $C_{1}=X \cup Y, C_{2}=Y \cup Z$ and $C_{3}=X \cup Z$. We can arrive at a contradiction by applying the above remark to $C_{1} \cap I, C_{2} \cap I$ and $C_{3} \cap I$ as follows. Assume without loss of generality that $x_{2}<_{\mathcal{R}} y_{2}<_{\mathcal{R}} z_{2}$. Then, by the above observation applied to $C_{1} \cap I$, it must be the case that one of $a, b$ is between $x_{2}$ and $y_{2}$ in $<_{\mathcal{R}}$ while the other is not. Similarly, by applying the observation to $C_{2} \cap I$ and $C_{3} \cap I$, we get that exactly one of $a, b$ is between $y_{2}$ and $z_{2}$ and exactly one of $a, b$ is between $x_{2}$ and $z_{2}$. It is easy to verify that simultaneously satisfying these three conditions is impossible.

Using the same techniques, we obtain the following result for $c-p$ - $\operatorname{Box}(1)$.

Lemma 7 Any $H^{l_{x}, l_{y}, l_{z}}$ graph such that $l_{z} \geq l_{y} \geq l_{x} \geq 2$ does not belong to $c$ - $p$-Box $(1)$.

The proof of this lemma follows the same ideas of the proof of Lemma 6 The main idea of the proof lies in the following remark.

Remark 3 Let $G$ be a graph and $\mathcal{R}$ be a c-p-Box(1)-realization of $G$. Consider any subset $S$ of vertices in $G$. Let $C$ be an induced cycle in $G$. From Lemma 3 , we can say that the linear order $<_{\mathcal{R}}$ restricted to $C \cap S$ partially respects $C$.

Proof of Lemma 7 Consider the set $S$ in the Remark 3 to be $\left\{a, b, x_{1}, y_{1}, z_{1}\right\}$. Following the lines of the proof of Lemma 6 , we can prove that there does not exist an order of the vertices in $S$ that respects the induced cycles $X \cup Y, Y \cup Z$ and $X \cup Z$.

With the previous Lemma we have presented an infinite family of graphs that do not belong $c-p$-Box(1). Nevertheless, some of these graphs do belong to $p$-Box $(1)$.

We shall see now that, indeed, the smaller cases $H^{2, l_{y}, l_{z}}$ and $H^{3, l_{y}, l_{z}}$ are minimal graphs that separate $p$ - $\operatorname{Box}(1)$ from $c-p$ - $\operatorname{Box}(1)$.

Lemma 8 Graphs $H^{2, l_{y}, l_{z}}$ and $H^{3, l_{y}, l_{z}}$ belong to $p$-Box(1) for any $l_{y}$ and $l_{z} \geq 2$ and $l_{y}$ and $l_{z} \geq 3$, respectively.

The proof of this lemma follows by giving orderings of the set of vertices of $H^{2, l_{y}, l_{z}}$ and $H^{3, l_{y}, l_{z}}$ that satisfy the four point condition for $p$-Box(1). Figure 7 shows graphically such orders.

We consider it important to stress the complete bipartite graph $K_{2,3}$ as a particular case of Lemma 7 and Lemma 8 i.e., $K_{2,3}$ belongs to $p$-BoX $(1)$ but it does not belong to $c$ - $p$-Box $(1)$. Such an importance 


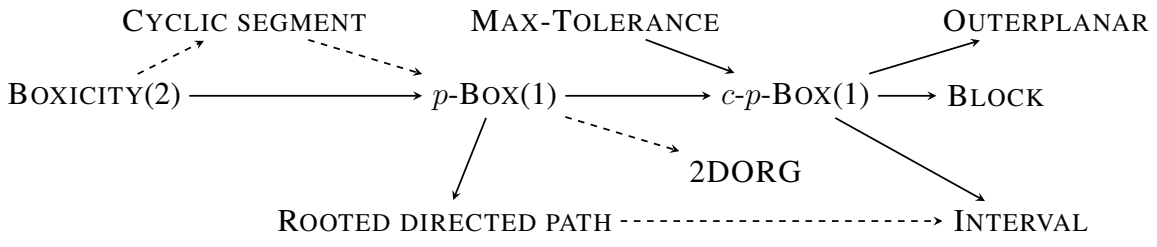

Fig. 8: Relation between the graph classes in the document. Arrows point from the superclass to the subclass. Dotted lines represent previous results and solid lines represent results proved in this document.

comes from the fact that $K_{2,3}$ is the smallest complete bipartite graph that does not belong to $c-p$-Box $(1)$. As a consequence of Lemma 7 and the fact that the property of belonging to $c-p$-Box $(1)$ is hereditary, we can say that any graph that contains a $H^{l_{x}, l_{y}, l_{z}}$ as an induced subgraph does not belong to $c-p$-Box (1). On the other hand, from Lemma 8 we know that some of these graphs do belong to $p$-Box(1).

\section{Future work}

Figure 8 places in context our results graphically. On the other hand, our work suggests several directions for future research. In our opinion, the most natural question is to find a combinatorial characterization for the $c-p-\operatorname{Box}(1)$ family. Another interesting open problem concerns to determine the complexity of the recognition problem for both $p$-Box(1) and $c-p$-Box(1) families. The study of higher dimensions of the families is an alternative way to continue this research. Another interesting question is the study of the family of graphs generated when points are embedded in a different topological space.

\section{Acknowledgements}

The authors would like to thank Antonio Fernández Anta and Marcos Kiwi because they are strongly involved in the origins of this study, even more, they contributed with enlightening ideas. We also thank the anonymous reviewer for his careful reading of our manuscript and his many insightful comments and suggestions.

\section{References}

[Bi192] T. Bilski. Embedding graphs in books: A survey. IEE Proceedings-E, 139(2):134-138, March 1992.

[BK79] F. Bernhart and P. C. Kainen. The book thickness of a graph. Journal of Combinatorial Theory, Series B, 27(3):320 - 331, 1979.

[BLS99] A. Brandstädt, V.B. Le, and J.P. Spinrad. Graph Classes: A Survey. Siam Monographs on Discrete Mathematics and Applications. Society for Industrial and Applied Mathematics, 1999.

[BM07] J.J.A. Bondy and U.S.R. Murty. Graph Theory. Graduate Texts in Mathematics Series. Springer London, 2007.

$\left[\mathrm{CCH}^{+}\right] \quad$ D. Catanzaro, S. Chaplick, S. Felsner, B. V. Halldórsson, M. M. Halldórsson, T. Hixon, and J. Stacho. Max point-tolerance graphs. Personal communication.

[CFS14] J. R. Correa, L. Feuilloley, and J. A. Soto Independent and Hitting Sets of Rectangles Intersecting a Diagonal Line. In Proceedings of the eleventh annual Latin American Theoretical INformatics Symposium., LATIN '14, pages 35-46, Montevideo, Uruguay, 2014. 
[EGP64] P. Erdös, A. W. Goodman, and L. Posa. The representation of a graph by set intersections, 1964.

[FCFM09] M. Farach-Colton, R. J. Fernandes, and M. A. Mosteiro. Bootstrapping a hop-optimal network in the weak sensor model. ACM Transactions on Algorithms, 5(4), 2009.

[GM82] M. C. Golumbic and C. L. Monma. A generalization of interval graphs with tolerances. Combinatorica, 1982.

[Gol04] M. C. Golumbic. Algorithmic Graph Theory and Perfect Graphs. Annals of Discrete Mathematics. Elsevier, 2004.

[GT04] M. C. Golumbic and A. N. Trenk. Tolerance Graphs. Cambridge Studies in Advanced Mathematics. Cambridge University Press, 2004.

[Gyö84] E. Györi. A minimax theorem on intervals. Journal of Combinatorial Theory, Series B, 37(1):1-9, 1984.

[Hix13] T. S. Hixon. Hook graphs and more: Some contributions to geometric graph theory. Master's thesis, Technische Universität Berlin, 2013.

[HK01] P. Hlineny and J. Kratochvíl. Representing graphs by disks and balls (a survey of recognition-complexity results). Discrete Mathematics, 229(1 - 3):101 - 124, 2001.

[KKLS06] M. Kaufmann, J. Kratochvíl, K. A. Lehmann, and A. R. Subramanian. Max-tolerance graphs as intersection graphs: cliques, cycles, and recognition. In Proceedings of the seventeenth annual ACM-SIAM symposium on Discrete algorithm, SODA '06, pages 832-841, New York, NY, USA, 2006. ACM.

[Koe36] P. Koebe. Kontaktprobleme der konformen abbildung. Berichte über die Verhand-lungen de Sächsischen, Akad. Wiss. Leipzig, Math.-Phys., 88:141-164, 1936.

[KWZ08] F. Kuhn, R. Wattenhofer, and A. Zollinger. Ad hoc networks beyond unit disk graphs. Wireless Networks, 14:715-729, 2008.

[Lub91] A. Lubiw. A weighted min-max relation for intervals. Journal of Combinatorial Theory, Series B, 53(2):151-172, 1991.

[MM99] T.A. McKee and F.R. McMorris. Topics in Intersection Graph Theory. Siam Monographs on Discrete Mathematics and Applications. Siam, 1999.

[Ola91] S. Olariu. An optimal greedy heuristic to color interval graphs. Information Processing Letters, 37(1):21 $-25,1991$.

[Rob69a] F. S. Roberts. Indifference graphs. In F. Harary, editor, Proof Techniques in Graph Theory, pages 139146. Academic Press, New York, 1969.

[Rob69b] F. S. Roberts. On the boxicity and cubicity of a graph. In W. T. Tutte, editor, Recent progress in combinatorics, pages 301-310. Academic press, New York, 1969.

[SMS45] S.-M. and E. Sur. Sur deux propriétés des classes d'ensembles. (french). Fundamenta Mathematicae, 33(1):303-307, 1945.

[Spi03] J.P. Spinrad. Efficient Graph Representations.: The Fields Institute for Research in Mathematical Sciences. Fields Institute Monographs, 19. American Mathematical Society, 2003.

[Tuc72] A. Tucker. A structure theorem for the consecutive 1's property. Journal of Combinatorial Theory, Series B, 12(2):153-162, 1972.

[WG86] M. Waterman and J. Griggs. Interval graphs and maps of dna. Bulletin of Mathematical Biology, 48:189195, 1986. 